\title{
Correction to: Lifelong Technology-Enhanced Learning
}

Viktoria Pammer-Schindler, Mar Pérez-Sanagustín, Hendrik Drachsler, Raymond Elferink, and Maren Scheffel

\section{Correction to:}

V. Pammer-Schindler et al. (Eds.):

Lifelong Technology-Enhanced Learning, LNCS 11082, https://doi.org/10.1007/978-3-319-98572-5

In the original version of this paper, the affiliation of the third editor was not correct. This has now been rectified. 